\title{
The effects of spatial frequency, orientation, and color upon binocular rivalry and monocular pattern alternation
}

\author{
FREDERICK L. KITTERLE and JOSEPH THOMAS \\ University of Toledo, Toledo, Ohio 43606
}

\begin{abstract}
The purpose of this investigation was to determine whether or not monocular and binocular rivalry are mediated by the same mechanism. The question was approached by examining, in a series of two experiments, the spatial frequency and orientation tuning characteristics for both forms of rivalry, as well as the effects of color upon monocular and binocular rivalry. The results of Experiment 1 indicate that binocular rivalry is insensitive to spatial frequency and color. In Experiment 2, the effects of spatial frequency and orientation were examined. The results of this experiment indicate that binocular rivalry is less sensitive to changes in orientation than is monocular alternation. There is no effect of color upon binocular rivalry. It was concluded that monocular alternation and binocular rivalry are mediated by different mechanisms.
\end{abstract}

When two optically superimposed grating targets that differ in orientation or spatial frequency are simultaneously presented to one eye or presented one to each eye, they appear to alternate. The former condition gives rise to monocular pattern alternation, the latter, to binocular rivalry.

The occurrence of monocular alternation is not found until one stimulus differs from the other by \pm 1 octave in spatial frequency of $\pm 10-15 \mathrm{deg}$ in orientation; it appears that monocular pattern alternation may reflect an antagonistic interaction (presumably involving lateral inhibition) between these channels. Since stimulus differences in spatial frequency and orientation are also required for binocular rivalry, it may be that the same mechanism that underlies monocular alternation underlies binocular rivalry. Wade (1975) found that there were phenomenal differences between the two types of rivalry. The number of alternations was considerably greater for binocular rivalry. An analysis of the dominance times of either grating (i.e., horizontal or vertical) or both together (composite) suggest that the temporal characteristics of the underlying mechanism differ in each case, implying that monocular pattern alternation and binocular rivalry may result from different mechanisms. The present study was designed to test this conclusion.

\section{EXPERIMENT 1: SPATIAL FREQUENCY}

Kitterle, Kaye, and Nixon (1974) showed that monocular pattern alternation is rather sensitive to spatial frequency differences between the two orthogonal gratings. A vertical grating was held at a constant spatial frequency; the spatial frequency of alternation was an inverted U-shaped function of the spatial fre- quency of the horizontal grating, with maximal rivalry when both gratings were 5 cycles/deg. This paradigm was used to compare the sensitivites of both types of rivalry with changes in spatial frequency.

In addition, comparisons were made between monocular pattern alternation and binocular rivalry when the components of the pattern were achromatic or of complementary color. It has been shown that monocular pattern alternation is greater for opponently colored patterns than for achromatic patterns but that rate of binocular rivalry is similar for colored and achromatic patterns (Wade, 1975). It is not clear, however, whether this apparent interaction between type of alternation and color of the pattern changes over spatial frequency.

\section{Method}

Subjects. Ten undergraduates at the University of Toledo served as observers in this experiment. All observers had 20/20 or corrected-to-20/20 vision with no astigmatism or color defects and were naive about the purpose of this study.

Apparatus. The stimuli, which were presented in a twochannel optical system, were photographic transparencies of sine-wave gratings. At a viewing distance of $76.5 \mathrm{~cm}$, the spatial frequencies of the gratings were $2.74,3.83,4.61,7.74$, and $10.96 \mathrm{cycles} / \mathrm{deg}$. The gratings were seen within a circular aperture subtending $1.5 \mathrm{deg}$ at the eye. The contrast of the gratings was .6. The adapting field luminance and space average luminance of the gratings were $.35 \log \mathrm{fL}$.

Procedure. Subjects were randomly assigned to one of the two experimental conditions (red-green, black-white) of five subjects each. Initially, the subject was dark adapted for $5 \mathrm{~min}$ and light adapted to the adapting field. During this time, subjects were told that they would view a circular aperture containing horizontal and vertical lines. They were told to report the distinctiveness of the lines by depressing one of three telegraph keys. One key was for the horizontal lines, another key was for the vertical lines, and a third key was to be depressed when the vertical and horizontal lines were equally distinct.

A trial consisted of the presentation of the pattern for $60 \mathrm{sec}$, 


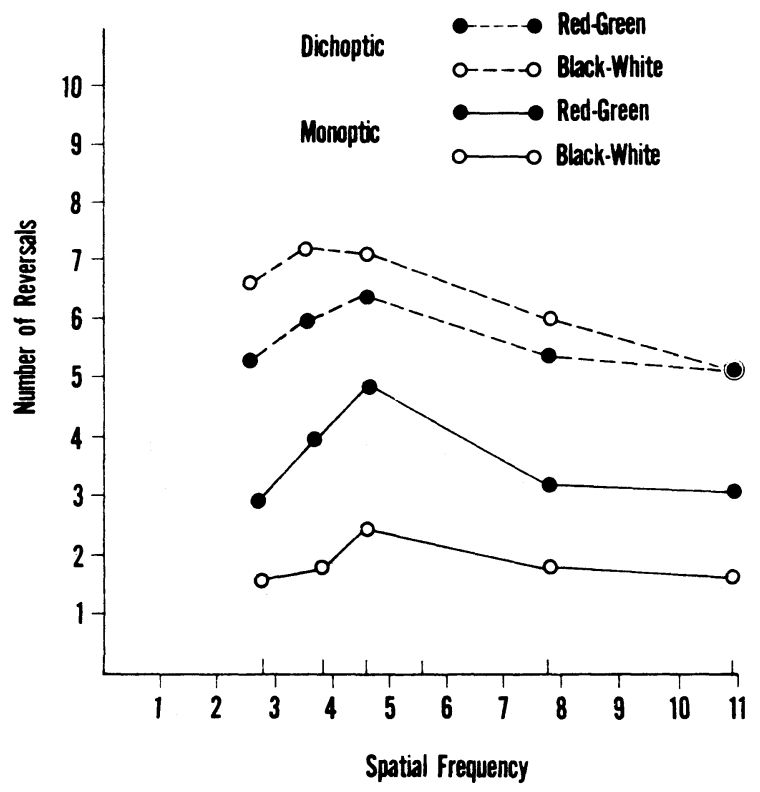

Figure 1. The number of reversals per minute are plotted as a function of the spatial frequency of the varied grating, averaged over components. The parameters of the curve are mode of viewing and color of the gratings.

with $15 \mathrm{sec}$ between trials. Each subject made five judgments. The spatial frequency of the horizontal grating was 4.61 cycles/ deg. Wratten filters Numbers 33 and 61 were used to produce the colors of red and green.

\section{Results}

In Figure 1, the number of keypresses are plotted as a function of spatial frequency of varied grating; the parameters of this figure are viewing conditions and color of the component gratings. Here, the data have been averaged over component keypress.

Under monoptic viewing, there were significantly more reversals for chromatic than for achromatic patterns $[F(1,8)=6.87, p<.05]$. These results confirm those reported by Rauschecker, Campbell, and Atkinson (1973). On the other hand, wavelength had no effect for dichoptic viewing $[F(1,8)=.14, p>.05]$. The interaction between color and spatial frequency was significant for monotopic viewing $[F(4,32)=3.09, p<.05]$, but not for binocular rivalry $[\mathrm{F}(4,32)=1.88, \mathrm{p}>.05]$. With chromatic stimuli, the monocular alternation curve has a more defined inverted $U$ shape, consistent with earlier results (Kitterle et al., 1974). Spatial frequency was significant for both modes of viewing [monocular, $\mathrm{F}(4,32)=8.97, \mathrm{p}<.01 ;$ dichoptic, $\mathrm{F}(4,32)=3.77$, $\mathrm{p}<.01]$. It is quite apparent from Figure 1 that the binocular rivalry curves (broken lines) are much broader over the spatial frequency range than are the monocular curves.

\section{Discussion}

These results indicate that the mechanism underlying alternation is more sensitive to the difference between the spatial frequencies that compose the pattern than is the mechanism underlying binocular rivalry (see Blake \& Fox, 1974).

In summary, the present experiment clearly indicates that binocular rivalry and monocular pattern alternation are mediated by different mechanisms. The former mechanism appears stronger and relatively insensitive to changes in either color or spatial frequency.
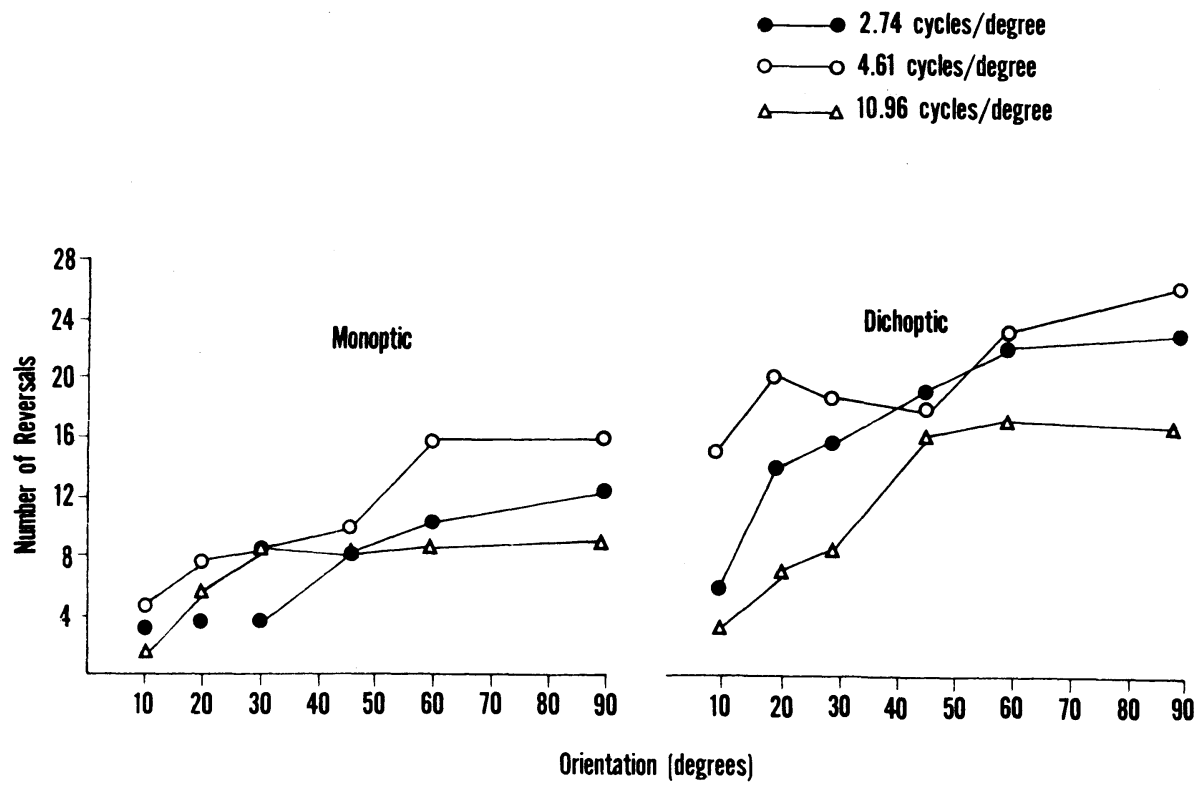

Figure 2. The number of reversals per minute are plotted as a function of the orientation of the varied grating. The parameters of the curve are mode of viewing and spatial frequency. 


\section{EXPERIMENT 2: EFFECT OF ORIENTATION}

There appears to be a difference in the way orientation influences monocular alternation and binocular rivalry. Monocular pattern alternation increases continuously as the angular separation between a vertical and variable grating increases up to $60 \mathrm{deg}$ (Campbell, Gilinsky, Howell, Riggs, \& Atkinson, 1973). Wade (1975) found that binocular rivalry was insensitive to orientational differences. This experiment compares both kinds of rivalry with both chromatic and achromatic stimulus patterns.

\section{Method}

Fifteen undergraduates served as observers in this experiment. There were three independent groups, in which the sinewave grating in the vertical orientation was of fixed spatial frequency $(2.74,4.61$, or 10.96 cycles/deg). The other grating was varied over the following orientations: $10,20,30,45,60$, and $90 \mathrm{deg}$. One grating was red and the other, green. The 15 subjects were randomly assigned to one of the three spatial frequency groups of 5 subjects each.

\section{Results}

The results of an analysis of variance upon these data indicate that spatial frequency, type of rivalry, and orientation significantly interacted $[F(10,60)=2.30$, $\mathrm{p}<.05]$. These results are presented in Figure 2. The number of reversals was significantly greater for binocular rivalry than for monocular alternation $[F(1,12)=$ $31.69, \mathrm{p}<.01$ ]. In addition, orientation had a significant effect for monocular alternation $[F(5,60)=11.22$, $\mathrm{p}<.01]$. The number of alternations increased as the varied grating approached $90 \mathrm{deg}$. Notice that the monocular data seem to asymptote sooner than the binocular data.

\section{Discussion}

The purpose of this experiment was to determine the effects of orientation upon both types of rivalrous phenomena. It was hypothesized that binocular rivalry would be insensitive to orientation. It appears, however, that binocular rivalry increases with changes in orientation. The apparent orientational sensitivity for binocular rivalry may be due, in part, to either cyclofusional or central fusional processes within the orientational range of 10-20 deg and possibly $30 \mathrm{deg}$ (Kertesz \& Jones, 1970). Due to partial or total fusion, reversals would decrease at the smaller orientation differences, thereby resulting in similar curves for monocular alteration and binocular rivalry.

This hypothesis receives additional support in an ancillary study in which comparisons were made between chromatic and achromatic stimuli under binocular rivalry. Differences in reversals were considerably greater in the range of $10-30 \mathrm{deg}$ for the achromatic stimuli than for color gratings. Fusion was facilitated by stimulus similarity, namely, achromaticity.

It thus appears that monocular pattern alternation and binocular rivalry are mediated by different mechanisms.

\section{REFERENCES}

Blake, R., \& Fox, R. Binocular rivalry suppression. Vision Research, 1974, 14, 1-5.

Campbell, F. W., Gilinsky, A. S., Howell, E. R., Riggs, L. A., \& AtKinson, J. The dependence of monocular rivalry on orientation. Perception, 1973, 2, 123-125.

Fox, R., \& RASCHE, F. Binocular rivalry and reciprocal inhibition. Perception \& Psychophysics, 1969, 5, 215-217.

Kertesz, A. E., \& Jones, R. W. Human cyclofusional response. Vision Research, 1970, 10, 891-896.

Kitte rle, F. L., KAYe, R. S., \& Nixon, H. Pattern alternation: Effects of spatial frequency and orientation. Perception \& Psychophysics, 1974, 16, 543-547.

Rauschecker, J. P. J., Campbell, F. W., \& Atkinson, J. Color opponent neurons in the human visual system. Nature, 1973, 245, 42-43.

WADE, N. J. Monocular and binocular rivalry between contours. Perception, 1975, 4, 85-95.

(Received for publication October 30, 1980.) 